\title{
New coronavirus with "pandemic potential" sparks global surveillance efforts
}

A novel coronavirus ( $\mathrm{nCoV}$ ) with what one Canadian researcher calls a "very alarming" death rate has triggered rapid research efforts to determine where it came from and where it may strike next. Two Canadian research teams are among those involved in the global response.

As of Mar. 26, the World Health Organization (WHO) had been informed of 17 confirmed cases since April 2012, with nine resulting deaths (for a mortality rate of 56\%). Most cases (11) were in Saudi Arabia, Qatar, United Arab Emirates and Jordan, although three were in the United Kingdom. The primary UK patient had recently travelled to Saudi Arabia and Pakistan, and was the source of infection for two people in his household, confirming human-to-human transmission of the virus.

Canada, however, is currently at low risk for the virus, says Dr. Kamran Khan, an infectious disease physician and scientist at St. Michael's Hospital in Toronto, Ontario. Still, he says, 'It's fair to say that this has pandemic potential. Whether it will evolve into one depends on many things."

Khan says two aspects could lead to a pandemic: human-to-human transmission and the possibility that cases have escaped detection to date. "We might be looking at the tip of the iceberg, only seeing those with serious forms [of the disease]. Others may have milder forms but might be capable of transmitting the disease to others."

"We don't yet know the course whether it will continue at a slow pace, accelerate or fizzle out." One of the factors affecting the course is international travel patterns, which played a major role in the severe acute respiratory syndrome (SARS) epidemic.

Khan and his team studied the risk of infection from Saudi Arabia during the H1N1 influenza pandemic, focusing on the annual pilgrimage to Mecca, which brings 1.7 million international

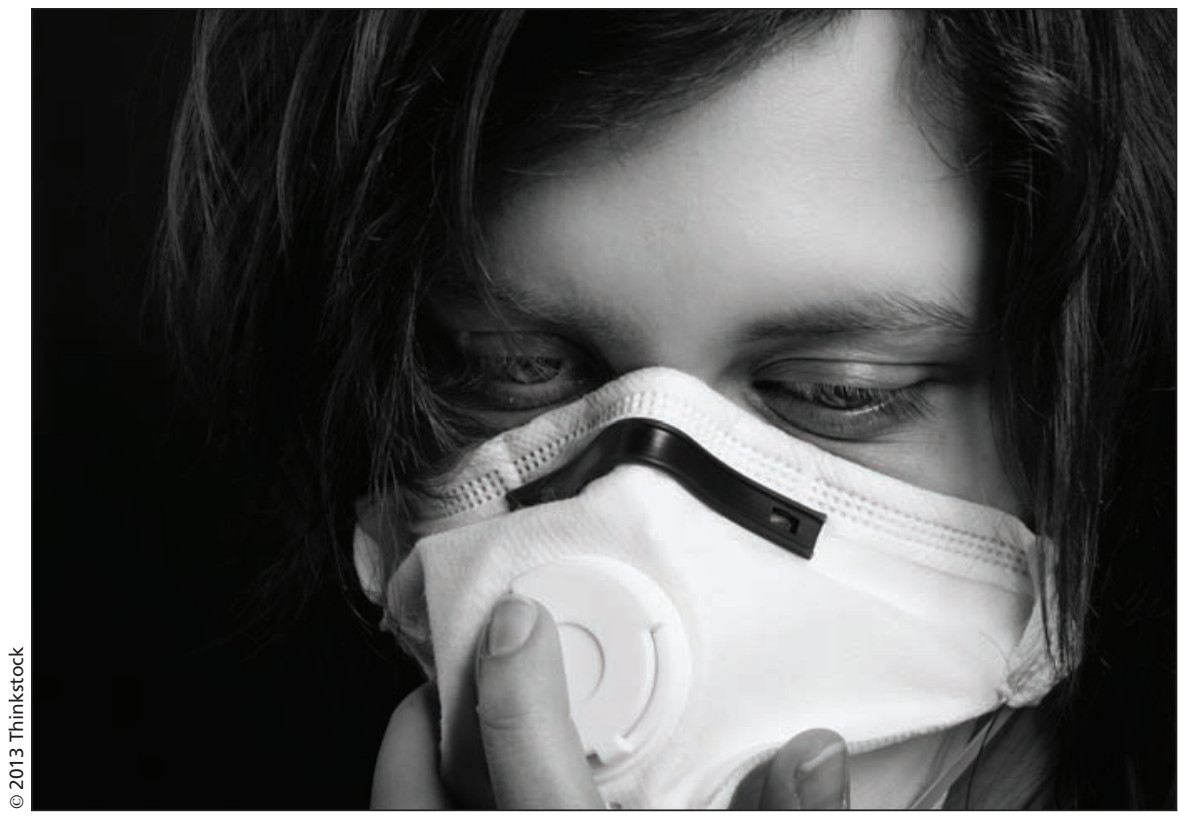

Canada is currently at low risk for the novel coronavirus, but that could change.

pilgrims to the country ( $J$ Travel Med 2010;17:75-81). Khan's figures indicate that Canada receives only $0.5 \%$ of travellers leaving the Saudi Arabia region, and the United States only $2 \%$.

Of more concern is that $25 \%$ of travellers leaving the Saudi Arabia region travel to India, Pakistan and Bangladesh, and $13 \%$ travel to Egypt. "Countries that are low-income, developing and without robust public health systems receive a large proportion of the travel," explains Khan. "The thinking right now is that main source areas [for $\mathrm{nCoV}$ ] are those three [Middle Eastern] countries. So the risk is low. If, however, we have cases circulating in other areas that we don't know about, the risk assessment is quite different."

Khan is working with a group called HealthMap, based at Children's Hospital Boston and Harvard Medical School in Massachusetts, to monitor international Internet "chatter" about infectious diseases as a type of "early warning system" about disease outbreaks.

David Kelvin, senior scientist at Toronto General Research Institute in
Ontario, agrees that surveillance is essential. An expert in emerging infectious diseases with research programs in Canada, China and Europe, Kelvin is working with international colleagues to set up surveillance studies in four global regions to screen for coronaviruses and determine infection rates.

"Without a rigorous surveillance program, we do not know the entire number of infections. Therefore, we do not know the denominator for calculating the true mortality rate." Nevertheless, the fact that 9 cases have resulted in death is "very alarming," Kelvin says.

Kelvin's team has studied the immune response to the SARS coronavirus and vaccination in an animal model (PLoS One 2012;7:e45842) and is currently working on a potential vaccine for $\mathrm{nCoV}$. In the quest for a vaccine, the discovery of the likely receptor for $\mathrm{nCoV}$ published Mar. 14 (Nature 2013;495:251-4) "is an important piece of the puzzle," explains Kelvin. While most coronaviruses cause only mild colds in humans, SARS and $\mathrm{nCoV}$ bind to cells deep within the 
lungs, leading to pneumonia or acute respiratory distress syndrome.

The $\mathrm{nCoV}$ receptor is found in the lungs of humans and also of other mammals, explaining how the disease has "jumped" from one species to others. A recent study found that $\mathrm{nCoV}$ is most closely related to two coronaviruses in bats in China and Japan (Emerg Microbes Infect 2012;1:e35). SARS has also been linked genetically to a bat coronavirus (PLoS One 2012; $7: e 45842$ ), thought to have "jumped" to civet cats and then from civet cats in Chinese markets to humans. nCoV may be infecting humans directly from bats or from another "intermediate" host.

"Most viruses over time cause only very mild symptoms in the natural host," explains Kelvin. "The reason for this is evolutionary - efficient transmission relies on relatively healthy individuals. I suspect the high mortality rate in humans is because the $\mathrm{nCoV}$ is adapted to bats, and when it jumps to humans it can cause severe disease."

Finding out how the patients acquired the disease, through contact with exotic species or humans, is key to understanding this outbreak, he says.

Khan says Canadian physicians can play a role in tracking the disease by being alert to possible cases. "From a public health perspective, the community has to be thinking of the worstcase scenario," he says.
The Public Health Agency of Canada and the US Centers for Disease Control (CDC) have both published notices to physicians about nCoV. CDC advises that patients with severe acute lower respiratory illness within 10 days after travelling from the Arabian peninsula or neighbouring countries should be evaluated.

Khan agrees, saying diagnosis is "actually quite challenging when you have a severe undifferentiated respiratory illness. After excluding all the usual suspects, we should check into recent travel and contacts with others with severe illness." - Carolyn Brown, Ottawa, Ont.

CMAJ 2013. DOI:10.1503/cmaj.109-4453 